\title{
CART POSITION CONTROL OF LINEAR INVERTED PENDULUM (LIP) USING INTELLIGENT TECHNIQUES
}

\author{
Soundarya M S, \\ Department of EIE \\ School of EEE \\ SASTRA UNIVERSITY \\ Thanjavur, India, 613401 \\ Jenitha Rashmi P \\ Department of EIE \\ School of EEE \\ SASTRA UNIVERSITY \\ Thanjavur, India, 613401
}

\author{
Akilaa Sre R \\ Department of EEE \\ School of EEE \\ SASTRA UNIVERSITY \\ Thanjavur, India, 613401 \\ Uma $S$ \\ Assistant Professor, Dept. of EIE \\ School of EEE \\ SASTRA UNIVERSITY \\ Thanjavur, India, 613401
}

\begin{abstract}
Linear Inverted Pendulum (LIP) System is a classical and bench mark problem in robotics and in many other applications in control systems. A Linear Inverted Pendulum (LIP) system essentially deals with two challenges as stabilizing a pendulum which is highly nonlinear in nature and controlling the cart position. Like the pendulum angle control, the Cart Position Control is also a quite potential challenging task. It is challenging because of the fact that the wheeled cart adds to the condition for tracking of the trajectory. Hence it is essential to track the required trajectory of the cart in a LIP system. This study deals with the trajectory control of Cart of a LIP model. A laboratory scale LIP has been considered the entire analysis and for which intelligent fuzzy, self-tuned fuzzy PID and GA tuned PID controllers are designed and are implemented in real time. In order to have a fair comparison, the real time results are compared with simulation results and a quantitative comparison has been carried out with IAE and ISE as the performance indices.
\end{abstract}

Keywords: Linear Inverted Pendulum, Intelligent fuzzy, Selftuned fuzzy PID, GA based PID

\section{Introduction}

Unstable systems are quite challenging for control applications. LIP system is a typical example for such unstable system in which the trajectory tracking plays an important role in robotics and in many other applications, irrespective of tracking, how fast the tracking is accomplished plays a vital role. In the literature many researchers have carried out research in this field. Ghosh et al. [3], Dongale et al. [2], ArpitJain et al. [1]. Though many researchers have carried out research in this field, many work deals with the conventional controller Satishkumar and Chidambaram. [6] as well as intelligent controllers Vaishnav and Khan, [10], Shikha Rao et al. [8], Tushar Upalanchiwar and Sakhare, [9], Roshan Kumar et al. [5], Sandeep Kaur and Gurpreet Bharti,[7]. In certain literature the modeling has been carried out with state model and modern controllers like LQR has been discussed. This work combines the features of conventional PID and intelligent techniques. Intelligent control design needs the understanding of the dynamics of the system. Intelligent method chosen for control is Fuzzy Logic Control (FLC). Optimization is achieved through Genetic Algorithm (GA) for optimizing the PID controller parameters. This study focuses on Cart Position Control of Linear Inverted Pendulum. PID controllers are the most common and widely utilized controllers because of its simplest structure and its easy implementation in most of the industrial applications. The control by PID makes the designer with the simple and least efforts on understanding the dynamics since it contributes much on mathematical part. The paper discusses mathematical model for cart position of Linear Inverted Pendulum in section 2, the various controller design in section 3 followed by simulation results in section 4 , experimental results in section 5 and quantitative analysis in section 6 and finally conclusion in section 7 .

\section{Process Description}

The pictorial view of the laboratory scale Linear Inverted Pendulum considered is shown in figure 1 .

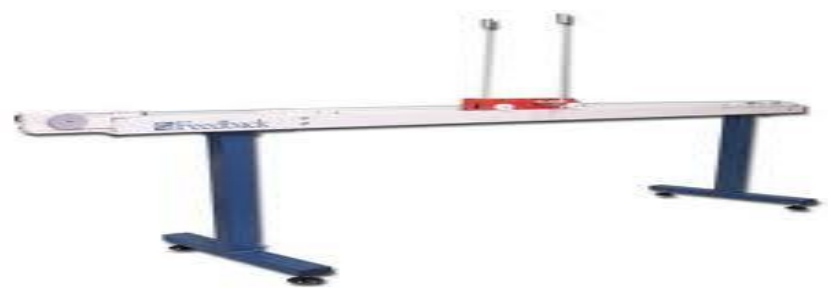

Fig. 1. Pictorial view of the Linear Inverted Pendulum setup The parameters of the laboratory scale Linear Inverted Pendulum system are: $F$ is the applied force to the cart, $x$ is the position of the cart, $M$ is the mass of the cart $(2.4 \mathrm{~kg}), m$ is the mass of the pendulum $(0.23 \mathrm{~kg}), L$ is the length of the pendulum $(0.38 \mathrm{~m}), I$ is the moment of inertia of the pendulum $\left(0.099 \mathrm{~kg}-\mathrm{m}^{2}\right), b$ is the coefficient of friction $(0.055 \mathrm{Ns} / \mathrm{m})$ and $g$ is the acceleration due to gravity $(9.81$ $\mathrm{m} / s^{2}$ ). The LIP setup is computer controlled and PCI cards are used for feedback signal information. The dynamics of the system can be expressed mathematically where $X(s)$ is the cart position and $U(s)$ is the controller output

$\frac{X(s)}{U(s)}=\frac{\left(I+m l^{2}\right) s^{2}-m g l}{\left((M+m)\left(I+m l^{2}\right)-m l^{2}\right) s^{4}+\left\{b\left(I+m l^{2}\right\} s^{3}-(M+m) m g l s^{2}-m g l b s\right.}$ Substituting all the above parameters and neglecting the friction coefficient $b$ (very small compared to other parameters) and the resultant transfer function is given below

$$
\frac{X(s)}{U(s)}=\frac{0.3894 s^{2}-2.6506}{s^{2}\left(s^{2}-6.807\right)} \approx \frac{0.3894}{s^{2}}
$$

The cancellation does not affect the internal stability problems, because the cancelled modes are available for feedback. D.C. motor converts the voltage $u$ to force $F$ and is represented by a gain block of 15 . Consideration of the gain block, the transfer function of the LIP for Cart Position Control is arrived as 


$$
\frac{X(s)}{U(s)}=\frac{5.841}{s^{2}}
$$

\section{Controllers Design}

The simple feedback block diagram with the process and the controller is shown in Figure 2, where $G_{p}$ is the transfer function of the process and $G_{c}$ is the transfer function of the controller.

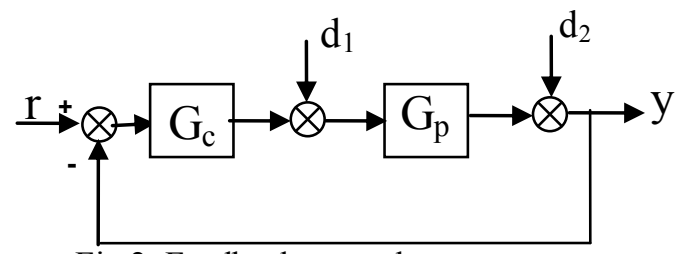

Fig.2. Feedback control structure

\section{Case (i) Fuzzy logic control}

Fuzzy Logic Control (FLC) is used to mimic the function of PID. The basic block diagram of a fuzzy controller is given in Figure 3. For the fuzzy controller, error and rate of error are considered as inputs and the output as controller output. The fuzzy inputs (error and rate of error) and the output (Controlled Output) are divided into 5 membership functions as Negative Big (BN), Negative (N), Zero (Z), Positive (P) and Positive Big (BP) and with these 25 rules are framed. The membership function considered is a triangular membership function. The rules framed for the Fuzzy Inference System (FIS) is given in table 1.

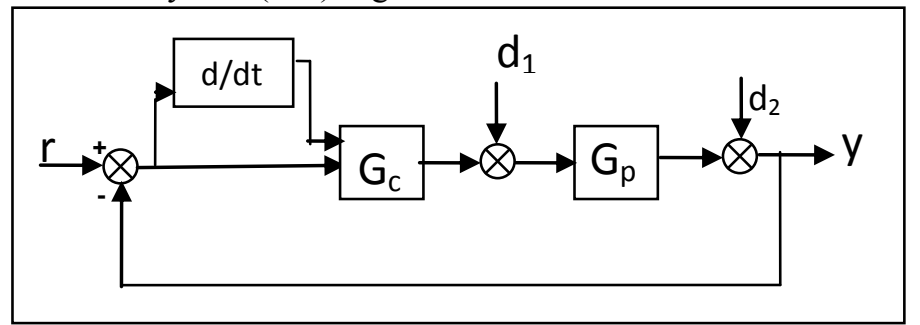

Fig.3. Basic block diagram of fuzzy controller

Table 1 Rule base table for FIS

\begin{tabular}{|c|c|c|c|c|c|}
\hline $\begin{array}{c}\text { Errorl Rate of } \\
\text { Error }\end{array}$ & BN & N & Z & P & BP \\
\hline BN & BN & BN & BN & N & Z \\
\hline N & BN & BN & BN & Z & P \\
\hline Z & BN & N & Z & P & BP \\
\hline P & N & Z & P & BP & BP \\
\hline BP & Z & P & BP & BP & BP \\
\hline
\end{tabular}

Case (ii) Self-tuned Fuzzy PID

The benefit of Fuzzy controller in combination with traditional PID makes the self-tuning more powerful. The factors of each parameter are tuned with fuzzy controller online every time during the process.

The basic block of a self tuned fuzzy PID is shown in Figure 4. The PID ranges are chosen from extreme values of conventional tuning methods. The fuzzy controller is designed with error and derivative error as input and the factors of each tuning parameters namely $k_{c}^{\prime}, k_{i}^{\prime}, k_{d}^{\prime}$ respectively (Zuraida Muhammad et al. [12]. The relation between these factors with the tuning parameters is expressed below

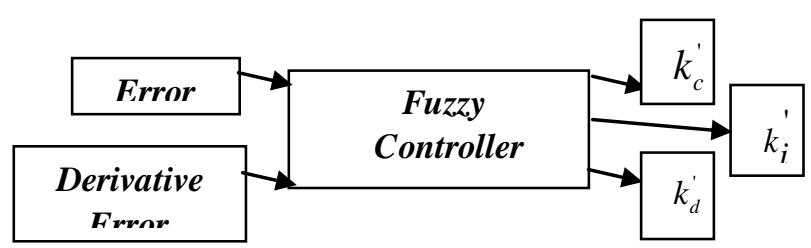

Fig. 4 Block Diagram of a Self-Tuned Fuzzy PID

$$
\begin{aligned}
& k_{c}^{\prime}=\frac{k_{c}-k_{c, \text { min }}}{k_{c, \text { max }}-k_{c, \text { min }}} \\
& k_{i}^{\prime}=\frac{k_{i}-k_{i, \text { min }}}{k_{\mathrm{i}, \max }-k_{i, \min }} \\
& k_{d}^{\prime}=\frac{k_{d}-k_{d, \min }}{k_{d, \text { max }}-k_{d, \text { min }}}
\end{aligned}
$$

\section{Case (iii) GA tuned PID}

PID controller equation considered is given below

$$
G_{c}=k_{c}\left(1+\frac{1}{\tau_{i} s}+\tau_{d} s\right)
$$

Tuning the PID parameter is optimized with GA technique. The objective function used is the performance index of the system. ITAE Integral Time Absolute Error is chosen as objective function. In general, reciprocal of the objective function is the fitness function. Selection function used is Roulette method and the input type is double vectored. The universe can be bounded according to the range required.

\section{Simulation results}

Simulation studies are carried out with the designed fuzzy, self tuned fuzzy and GA tuned PID controller. Multiple step input has been provided and the obtained closed loop responses are shown in figure 4 respectively. A unit step input at time $\mathrm{t}=0 \mathrm{sec}$ and another step of magnitude 0.5 at time $\mathrm{t}=50 \mathrm{sec}$ and another step input of magnitude 0.5 at time $\mathrm{t}=100$ has been provided and a negative output disturbance at time $\mathrm{t}=125 \mathrm{sec}$ of magnitude 0.1 is also provided and the obtained closed loop response is shown in Figure 5 respectively.

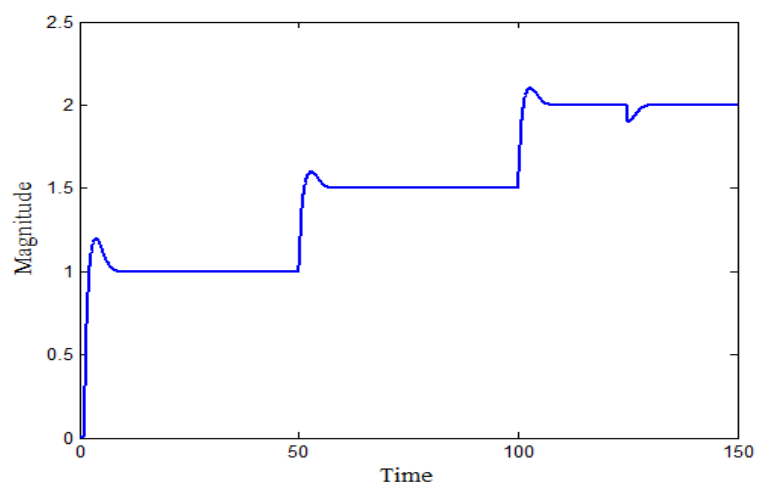

Fig . 5. Closed loop response for cart control of LIP with multiple step input using Fuzzy controller 


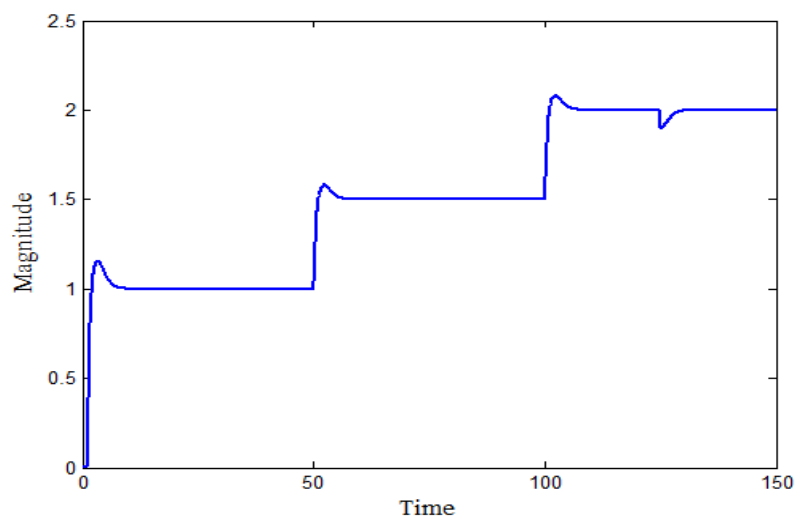

Fig. 6 Closed loop response of cart position for perturbed condition for a perturbation of $+30 \%$ in $\mathrm{k}_{\mathrm{p}}$

In order to show the robustness advantage of the fuzzy controller, perturbations are considered in the process gain and a perturbation of $+30 \%$ is considered in $\mathrm{k}_{\mathrm{p}}$ and the corresponding closed loop response is shown in Figure 6. A. Network components

In this study, the variable range of the parameters $\mathrm{K}_{\mathrm{p}}, \mathrm{K}_{\mathrm{i}}$ and $\mathrm{K}_{\mathrm{d}}$ varies in between $\left[\mathrm{K}_{\mathrm{pmin}}, \mathrm{K}_{\mathrm{pmax}}\right]$, [ $\left.\mathrm{K}_{\mathrm{imin}}, \mathrm{K}_{\mathrm{imax}}\right]$, [ $\left.\mathrm{K}_{\mathrm{dmin}}, \mathrm{K}_{\mathrm{dmax}}\right]$. The range of PID parameters are obtained from the simulation of PID controller to get the feasible and optimum performance [12] from which the range for each parameters are $\mathrm{k}_{\mathrm{p}}=\left[\begin{array}{ll}12 & 67\end{array}\right], \mathrm{k}_{\mathrm{i}}=\left[\begin{array}{ll}0 & 2.5\end{array}\right]$ and $k_{d}=\left[\begin{array}{ll}21 & 4\end{array}\right]$ respectively. With these values From the equation 4 the appropriate value of $\mathrm{k}_{\mathrm{p}}, \mathrm{k}_{\mathrm{i}}$ and $\mathrm{k}_{\mathrm{d}}$ are obtained as $k_{c}=55 k_{c}^{\prime}+12, k_{i}=2.5 k_{i}^{\prime}$ and $k_{d}=-17 k_{d}^{\prime}+21$ With these values the self tuned fuzzy controller simulation has been carried out. A step input of magnitude 0.5 at $t=1 \mathrm{sec}$ and another step input of magnitude of 0.5 and at time $t=50 \mathrm{sec}$ and another step input of magnitude of 0.5 and at $t=100 \mathrm{sec}$ and a negative disturbance at $\mathrm{t}=125 \mathrm{sec}$ of magnitude of 0.1 has been provided and the obtained closed loop response is shown in Figure 7 respectively. From the figure it is clear that self tuned fuzzy PID gives superior set point tracking with no overshoot and a good disturbance rejection.

In order to show the robustness advantage of the self tune fuzzy PID controller, perturbations are considered in the process gain and a perturbation of $+30 \%$ is considered in $\mathrm{k}_{\mathrm{p}}$ and the corresponding closed loop response is shown in Figure 8.

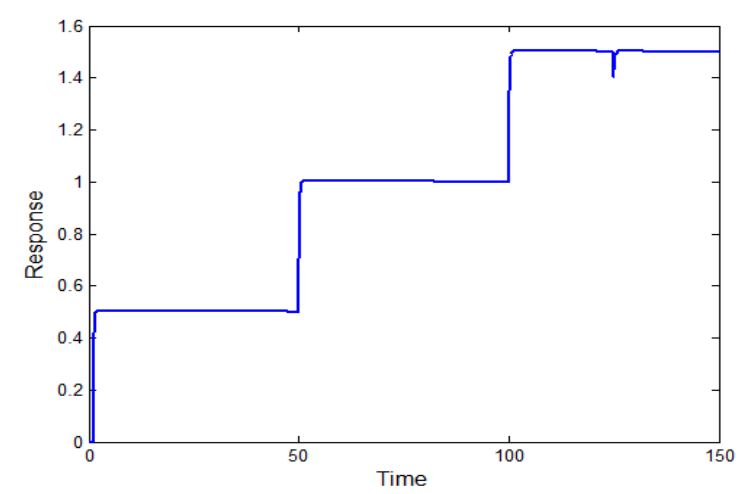

Fig. 7 Closed loop response of a self-tuned Fuzzy PID for cart position of LIP with multiple step inputs

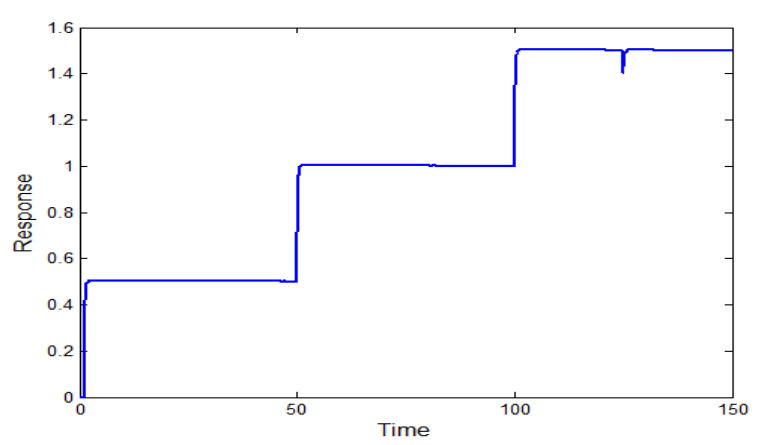

Fig. 8 Closed loop response of Cart Position Control with self tuned fuzzy PID for perturbed condition. Perturbation of $+30 \%$ in $\mathrm{k}_{\mathrm{p}}$.

With GA tuned fuzzy, the tuning parameters obtained are $\mathrm{k}_{\mathrm{p}}=$ $3.549, \mathrm{k}_{\mathrm{i}}=1.172$ and $\mathrm{k}_{\mathrm{d}}=1.421$. Figure 8 shows the closed loop response obtained for a GA based PID controller. A step input of magnitude 1 at time $t=1 \mathrm{sec}$ is considered and another step input of magnitude 0.5 has been considered at time $\mathrm{t}=20 \mathrm{sec}$ and $40 \mathrm{sec}$ respectively and the corresponding closed loop response is shown in Figure 9. It is clear from the response that optimized PID gives improved results.

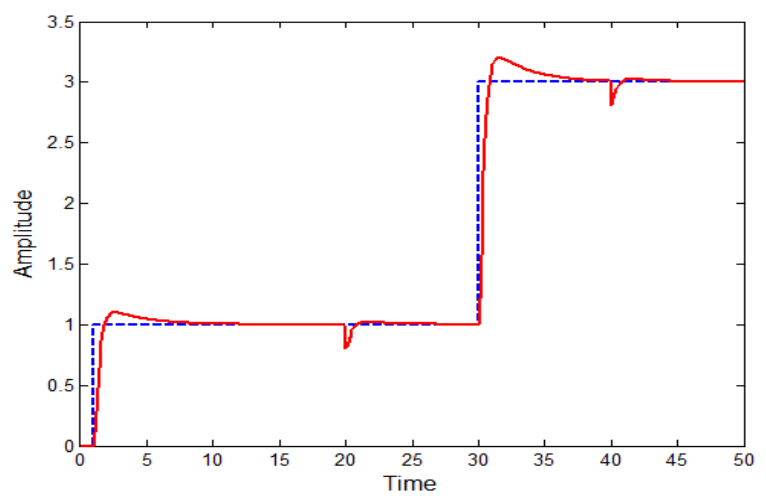

Fig. 9 Closed loop response for Cart Position Control of a GA based PID controller

\section{Experimental Results}

Experimentation has been carried out with the designed fuzzy, fuzzy PID and with GA tuned PID controllers. The system track length is $1 \mathrm{~m}$ and the cart is supposed to move for a distance of $0.4 \mathrm{~m}$ from the center point. The input to the actuator should be within $\pm 2.5 \mathrm{~V}$. The system consists of two degree of freedom, however the setup has been provided with only one DC motor as the only actuator in the system.

The system has been provided with 3 periodic sine waves as input of magnitude $0.2,0.15$ and 0.4 and of frequency 1,10 and 12 respectively. This in turn applied with the slider gain with minimum and maximum values namely 0.1 and 0.2 respectively. Further, it is saturated with upper and lower limits of \pm 0.35 with sample time of -1 as reference. The closed loop response obtained with fuzzy controller is shown in Figure 10.

In order to carry out a fair comparison, the results obtained in real time are compared with the simulation results.

IAE has been chosen as the performance index. The experimental results obtained with GA based PID is shown in Figure 12. 


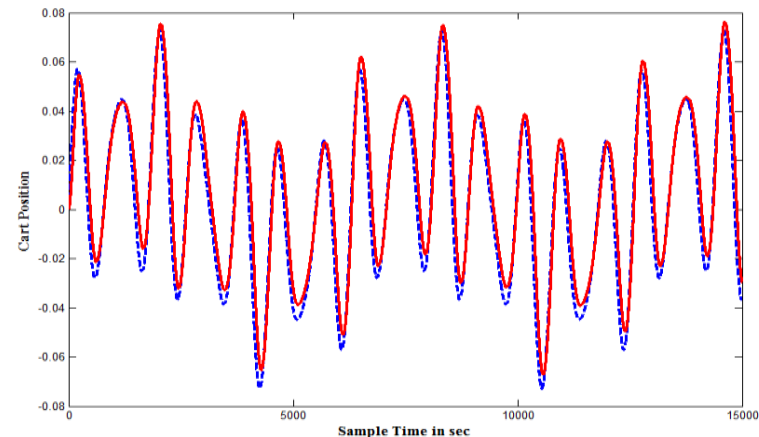

Fig. 10 Closed loop response of Cart Position Control of LIP using Fuzzy controller ( dash- Desired Input, solid line- Cart position)

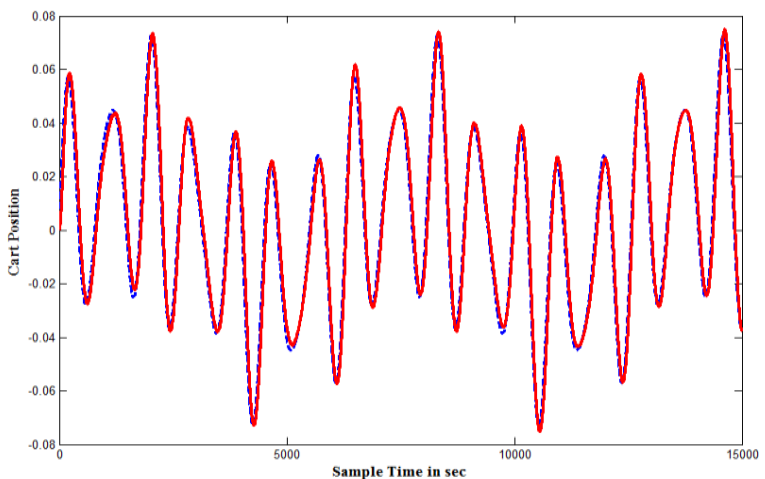

Fig. 11 Closed loop response of Cart Position Control using SelfTuned Fuzzy PID ( dash- Desired Input, solid line- Cart position)

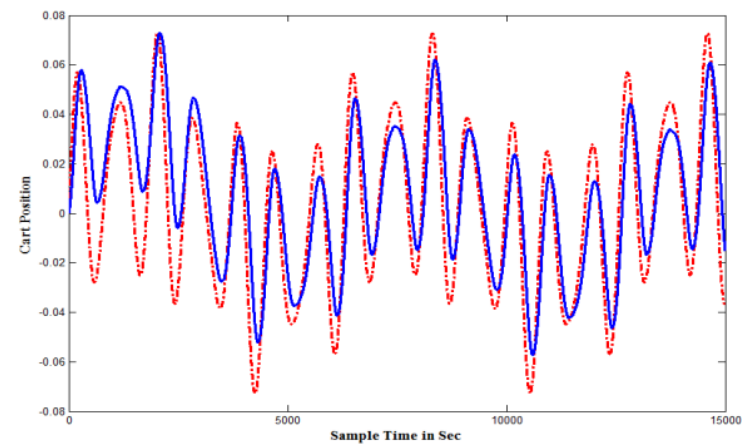

Fig. 12 Closed loop response of Cart Position Control using GA based PID

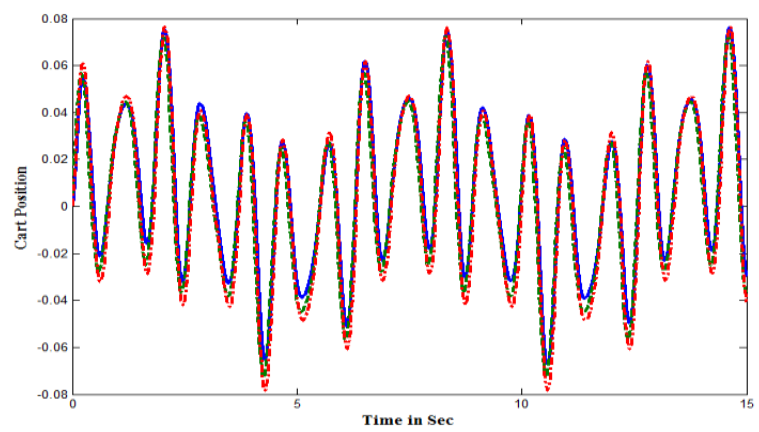

Fig.13 Closed loop response of Cart Position Control of LIP with fuzzy controller (dash- Desired input, Solid- Cart Position in real time, dash-dot- Cart Position in simulation)
Figure 13 shows the closed loop response obtained for Cart Position Control of LIP with designed fuzzy controller. From the figure it is clear that the real time results are very close to the result obtained in simulation. Figure 14 shows the closed loop response obtained for cart position of LIP with designed self tuned fuzzy PID controller. Figure 15 shows the closed loop response obtained for cart position of LIP with designed GA based PID controller.

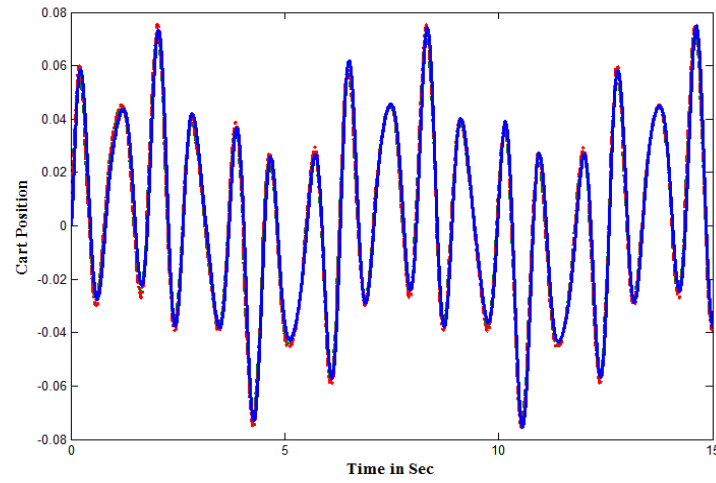

Fig. 14 Closed loop response of Cart Position of LIP with self-tuned fuzzy controller (Legend as given in figure 13)

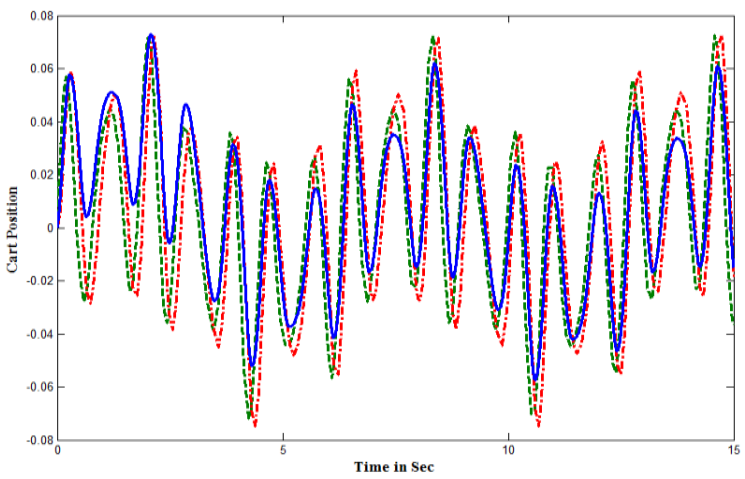

Fig. 15 Closed loop response of Cart Position Control of LIP for GA tuned PID(Legend as given in figure 13)

\section{Performance Analysis}

ISE, IAE, Rise time, settling time; overshoot are considered as performance measures for trajectory control for simulation results.

Table 2 Performance analysis of Cart Position Control for simulated results

\begin{tabular}{|c|c|c|c|}
\hline Parameters & $\begin{array}{c}\text { Fuzzy } \\
\text { PID }\end{array}$ & $\begin{array}{c}\text { Self -tuned } \\
\text { Fuzzy PID }\end{array}$ & $\begin{array}{c}\text { GA based } \\
\text { PID }\end{array}$ \\
\hline ISE & 0.027 & 0.053 & 0.689 \\
\hline IAE & 0.052 & 0.031 & 0.258 \\
\hline Rise time $t_{r}$ & 0.266 & 0.079 & 0.569 \\
\hline $\begin{array}{c}\text { Settling time } \\
t_{s}\end{array}$ & 3.779 & 1.132 & 6.421 \\
\hline $\begin{array}{c}\text { Overshoot } \\
M_{p}\end{array}$ & 83.09 & 0.038 & 9.243 \\
\hline
\end{tabular}

Acknowledgements

The experimental work has been carried out in the Advanced Process Control laboratory in SASTRA UNIVERSITY, 
Thanjavur, India with a prototype Linear Inverted Pendulum model.

\section{Conclusions}

The choice of the controllers is based on the required performance of the system. However use of fuzzy in control makes the system cheap and efficient. Based on the simulation results as well as the experimental results one can infer that self tuned fuzzy gives better results with fast set point tracking with no overshoot and with a good disturbance rejection than the GA tuned PID and a simple fuzzy controller.

\section{References}

[1]. ArpitJain, Deeptayal and NehaSehgal, Control of Non-Linear Inverted Pendulum using Fuzzy Logic Controller, International Journal of Computer Applications (0975 - 8887) 69 (37), 2013.

[2]. Dongale.T.D, Kulkarni.T.G, Kadam.P.A, Mudholkar.P.R, Simplified Method for compiling Rule Base Matrix, International Journal of Soft Computing and Engineering (IJSCE) , ISSN: 2231-2307, 2(1), 39-43, 2012.

[3]. Ghosh A, Krishnan T.R and Subudhi B, Brief Paper Robust proportional-integral-derivative compensation of an inverted cart-pendulum system: an experimental study, IET Control Theory and Applications, 2011.

[4]. Nour M. I. H., Ooi J and Chan K Y, Fuzzy Logic Control Vs. Conventional PID Control of an Inverted Pendulum Robot, International conference on Intelligent and advanced systems, 209-214, 2007.

[5]. Roshan Kumar, Ravi Bhushan Singh, Jayanta Das, Modeling and Simulation of Inverted Pendulum System using Matlab: overview, International Journal of Mechanical and Production Engineering, 1(4), 52-55, 2013.

[6]. Satishkumar.B and Chidambaram.M, Control of unstable bioreactor using fuzzy tuned PI controller, Bioprocess Engineering, 20(2), 127-132, 1999.

[7]. Sandeep Kaur and Gurpreet Bharti, Two Inputs Two Output Fuzzy Controller System Design using MATLAB, International Journal of Advances in Engineering, Science and Technology (IJAEST), 2(3), 209-218, 2012.

[8]. Shikha Rao, Lini Mathew \& Rahul Gupta, Performance Comparison of Fuzzy Logic Controller with Different Types of Membership Function using Matlab Tools, IR Net Transactions on Electrical and Electronics Engineering, 2011.

[9]. Tushar Upalanchiwar and Sakhare A.V, Design and implementation of the fuzzy PID controller using MATLAB/SIMULINK model, International Journal of Research in Computer and Communication Technology, 3(3), 369-372, 2014.

[10]. Vaishnav S.R. and Khan Z.J, Design and Performance of PID and Fuzzy Logic Controller with Smaller Rule Set for Higher Order System, Proceedings of the World Congress on Engineering and Computer Science 2007, San Francisco, USA, 2007.

[11]. Vikram Chopra, Sunil K. Singla \& Lillie Dewan, Comparative Analysis of Tuning a PID Controller using Intelligent Methods, Acta Polytechnica Hungarica, 11(8), 235$249,2014$.

[12]. Zuraida Muhammad, Zakiah Mohd Yusoff, Nurhani Kasuan, Mohd Noor Nasriq Nordin, Mohd Hezri Fazalul Rahiman, Mohd Nasir Taib, Online Tuning PID using Fuzzy Logic Controller with Self-Tuning Method, IEEE $3 r d$
International Conference on System Engineering and Technology, 94-98, 2013. 